\title{
Monitoring construction labour productivity by way of a smart technology approach
}

Eirini Konstantinou PhD

Department of Engineering, Laing O'Rourke Centre, Cambridge, UK (Orcid:0000-0003-3652-5557) (corresponding author: eirinikonstant@gmail.com)
Ioannis Brilakis PhD

Department of Engineering, Laing O'Rourke Centre, Cambridge, UK (Orcid:0000-0003-1829-2083)

Over the past decades, the construction industry lags further and further behind the manufacturing sector when productivity is considered. Current practices for managing labour productivity are labour intensive, time and cost consuming and error prone. They are mainly reactive processes initiated after the detection of a negatively influencing factor. Although research studies have been performed towards leveraging these limitations, a gap still exists in managing the labour productivity of multiple workers at the same time accurately, unobtrusively and cost and time efficiently. This paper proposes a trajectory-based method to address this gap. Firstly, a computer visionbased method extracts the three-dimensional (3D) trajectories of workers. Secondly, a clustering-based method converts the 3D trajectories into work cycles that depict the labour input. The proposed method features an accuracy of $84 \%$ in terms of inferring the total labour time spent on construction-related tasks - that is, direct work.

\begin{tabular}{|c|c|c|}
\hline \multicolumn{2}{|l|}{ Notations } & $x_{\min }$ \\
\hline$a_{x t_{k_{k}}}$ & inclination of the $X T$ time series & $X T$ \\
\hline$a_{y t_{k_{i}}}$ & inclination of the $Y T$ time series & $X Z$ \\
\hline$a_{z t_{k}}$ & inclination of the $Z T$ time series & $y_{\text {error }}$ \\
\hline centroid $_{\|\left.\vec{v}\right|_{x z_{\max }}}$ & maximum value of the cluster with the largest & $y_{\max }$ \\
\hline & speed values & $y_{\text {min }}$ \\
\hline centroid $_{\|\left.{ }_{i}\right|_{x z_{\min }}}$ & $\begin{array}{l}\text { minimum value of the cluster with the smallest } \\
\text { speed values }\end{array}$ & $Z$ \\
\hline$c_{i}$ & work cycle & $Z_{\text {crit }}$ \\
\hline$D$ & limit of error & $Z_{\text {error }}$ \\
\hline$d_{c_{i}}$ & duration of a work cycle & $Z_{\max }$ \\
\hline$d_{s}$ & time interval for partitioning the $3 \mathrm{D}$ trajectories & $Z_{\min }$ \\
\hline$d_{\text {stop' }}$ & duration of the semantic 'stop' event & $Z T$ \\
\hline$k$ & smoothing step & $\lambda$ \\
\hline
\end{tabular}

$k_{x t} \quad$ smoothing step of the motion along the $X$-axis

$k_{y t} \quad$ smoothing step of the motion along the $Y$-axis

$k_{z t} \quad$ smoothing step of the motion along the $Z$-axis

$N \quad$ total number of subtrajectories

$n \quad$ total number of clusters

$p \quad$ estimate of the accuracy of the test

$S_{x z} \quad$ group of subtrajectories

$s_{i} \quad$ single subtrajectory

$t_{\text {end }} \quad$ ending time of a semantic event's last

subtrajectory

$t_{\text {start }} \quad$ starting time of a semantic event's first subtrajectory

$T_{x z} \quad$ trajectory that depicts the motion of a worker across the floor plane

$\left\|\vec{v}_{i}\right\|_{x z} \quad$ speed value of a single subtrajectory

$\left\|\vec{v}_{x z}\right\| \quad$ group of speed values of subtrajectories

$w_{i} \quad$ weight of the weighted moving average method

$X \quad$ Cartesian coordinate in the direction along the $X$-axis

$x_{\text {error }} \quad$ smoothing error along the $X$-axis

$x_{\max }$ minimum smoothing error along the $X$-axis motion along the $X$-axis over time

floor plane Cartesian coordinates smoothing error along the $Y$-axis maximum smoothing error along the $Y$-axis minimum smoothing error along the $Y$-axis Cartesian coordinate in the direction along the Z-axis confidence level based on normal distribution smoothing error along the $Z$-axis maximum smoothing error along the $Z$-axis minimum smoothing error along the $Z$-axis motion along the $Z$-axis over time constant of the exponential moving average method

\section{Introduction}

The construction sector has gradually created a significant labour productivity gap compared with other industries over the past five decades. Data show that labour productivity in construction has not improved since 1964 (Teicholz, 2004; Teicholz, 2013). In fact, it is estimated that only $50 \%$ of the total construction time is productive (Horman and Kenley, 2005; Picard, 2004). Low labour productivity affects the revenue of the construction sector, considering that $33-50 \%$ of the entire cost of a typical project is spent on labour (Hanna et al., 2005). Such revenue loss also has a direct effect on the national economies as $9.0 \%$ of the gross domestic product of the European Union (EU) belongs to the construction industry sector (European Construction Industry Federation, 2019). The construction sector contributed $€ 1.427$ billion in 2018 (EU28) and supplied almost 6.4\% of Europe's total employment (14.8 million workers).

Previous studies identified the factors that affect labour productivity in a negative way (El-Gohary and Aziz, 2014; 
Gundecha, 2012; Jarkas and Bitar, 2012; Kuykendall, 2007; Lim and Alum, 1995; Makulsawatudom et al., 2004). These factors are mainly non-periodic phenomena that are directly related to onsite tasks, such as overtime, safety, resources, scheduling, rest areas, transportation, congestion, disruptions, skills, fatigue, absenteeism and unscheduled breaks. Therefore, managing such factors efficiently requires proactive monitoring. Productivity is defined as the ratio of output to input (Lim, 1996). The current state of practice in monitoring labour productivity is mainly based on manual observations and work sampling techniques (AMAC Consultants, 2004; Carrasco et al., 2013; Dozzi and AbourRizk, 1993; Shehata and El-Gohary, 2011). These practices can be grouped into two categories. The practices of the first category only indicate whether workers are 'productive/unproductive' or 'working/non-working'. Such practices are $(a)$ the $5 \mathrm{~min}$ rating that relies on the observations of tasks for a small period of time, (b) the field rating that calculates the fraction of productive workers over the total number of productive and unproductive ones to pinpoint whether something is wrong with a task's productivity and $(c)$ the work sampling that analyses a small sample of data to return a general assessment of workers' performance - for example, effective, contributory and noneffective. The practices of the second category provide more details. Such practices include $(a)$ the crew balance charts that illustrate each worker's performance over time using a stopwatch, (b) the field surveys that use questionnaires (e.g. foreman delay survey and craftsman questionnaire) to provide an understanding of the possible causes of bad performance, $(c)$ the photographic and video-based techniques that rely on human operators to review video data and still images and, lastly, $(d)$ the method productivity delay models, where observers report in forms the cycle time of a leading resource along with the causes of delays. The main limitation of all the above practices is that they are extremely labour-intensive and time-consuming considering that a large number of workers must be monitored in a manual way on a daily basis for the entire duration of their work shifts. For instance, an expert engineer needs on average $3.5 \mathrm{~h}$ to extract the productivity of a 14 min video capturing a worker while installing a scaffold (Gong and Caldas, 2011).

In summary, currently, it is not practically feasible to monitor labour productivity proactively. This is due to the manual effort of existing practices. This paper proposes a novel trajectory-based method to address this shortcoming. It extends the authors' previous work as presented in Konstantinou and Brilakis (2018a). The remainder of this paper continues with a discussion of the current state of research in automated productivity monitoring methods. Then, the proposed solution is presented and evaluated in the following two sections. In the last section, conclusions and future work are discussed.

\section{Current research on automated productivity monitoring methods}

Existing research on monitoring labour productivity can be divided into two categories. The studies of the first category convert the time workers spend at predefined work zones (e.g. concrete pouring zone, waiting and travelling) directly into labour input. Such studies are either tag-based or tagless. The tag-based studies rely on tags (e.g. global positioning system, radiofrequency identification and ultra-wideband) attached to workers and earthmoving equipment (Cheng et al., 2011; Cheng et al., 2013; Jiang et al., 2015; Navon and Goldschmidt, 2003; Sedehi, 2010). However, the purchase of tags imposes a regular cost (Nasr et al. 2013), while their physical attachment on workers causes a feeling of discomfort (Juels, 2006). On the other hand, tagless studies use non-obtrusive computer vision-based methods to track the location of workers within a camera's field of view (Bügler et al., 2014; Gong and Caldas, 2011). Still, such methods remain semiautomated as human operators are required to adjust the proposed process models to every worker (Gong and Caldas, 2011).

The second category consists of activity-based studies that detect and link activities (i.e. physical description of a subtask) such as bending, hammering and drilling to specific tasks to monitor productivity. The studies of this category use machine-learning algorithms, trained on physiological, posture and audio data to achieve this. Posture data were used for monitoring the productivity of both workers and earthmoving equipment (Bai et al., 2008; Khosrowpour et al., 2014; Zou and Kim, 2007). However, such data were proven insufficient for the case of workers due to the lack of distinguishable postures. The studies that rely on physiological data use heart and breathing rates, body force and angular rate. Although breathing rates were proven insufficient for monitoring individual's labour productivity (Gatti et al., 2014), the body's force and angular rate were successfully used for identifying multiple different activities such as sawing, turning a wrench and loading/unloading/pushing a wheelbarrow (Akhavian and Behzadan, 2016). The main limitation of all such studies is the requirement of attaching obtrusive physiological status monitoring and inertial measurement unit (Imu) sensors on workers. Lastly, the use of audio data is limited to tasks that generate unique sounds such as hammering and drilling and to only one type of task per instance (Cheng et al., 2017; Weerasinghe and Ruwanpura, 2010).

In summary, the prevalent approach to monitoring labour productivity exploits four types of trajectory data: $(a)$ walking path trajectories, (b) dense trajectories (posture), (c) physiological rates such as heart rate (beats/min) and respiratory rate (breaths/ $\min$ ) and (d) sound signals. Unfortunately, so far, none of the existing studies meet the requirements for proactive monitoring of labour productivity in an accurate, non-obtrusive and time- and cost-efficient way for multiple workers. This gap in knowledge exists as current studies are mainly tailored to specific tasks. The main aim of the research presented in this paper is to propose a fully automated method for monitoring labour productivity of multiple construction workers in a non-labour-intensive and accurate way. The main objectives of this paper are $(a)$ to design a method that monitors the activities of construction workers 
unobtrusively, $(b)$ to devise a method that is invariant to the large variety of construction tasks and $(c)$ to develop a method that groups trajectories of workers into clusters of similar pattern that depict labour input.

\section{Proposed solution}

This section presents a method that converts workers' walking path trajectories into labour input. As previously mentioned, labour productivity is calculated by dividing the total labour output over the total input. The proposed method focuses on the input as the monitoring of the output is quite straightforward through visual inspection at the end of work shifts (e.g. number of steel cages prepared and metres of brick wall painted). Figure 1 illustrates the overall proposed method. The skewed parallelogram shapes refer to processes and the circular to inputs/outputs. The solid-line-shaped skewed parallelograms depict methods of novel contribution, while the dashed skewed parallelograms are methods taken from literature.

Firstly, the work cycles of construction workers are detected regardless of the type or the number of tasks they perform. In construction, a work cycle is defined as the total time a worker spends on a task (Dozzi and AbourRizk, 1993). The initial inputs of the proposed method are the three-dimensional (3D) trajectories of workers, which are extracted by a computer visionbased 3D tracking method (Konstantinou and Brilakis 2018b; Konstantinou et al. 2019). The 3D tracking trajectories depict the movements of the centroid of a bounding box that captures a worker's body. A trajectory $\left(T_{x z}\right)$ depicts the motion of a worker

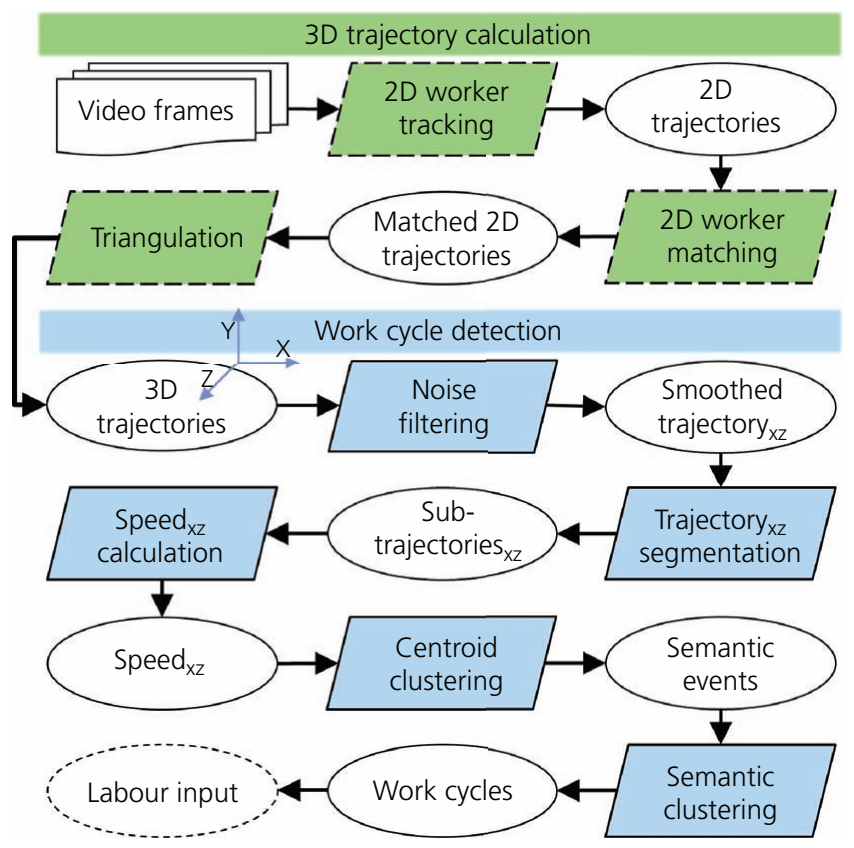

Figure 1. Flowchart of the proposed method for monitoring construction workers' labour productivity across the floor plane $(X Z)$ over time. This motion is described by the $X$ and $Z$ Cartesian coordinates. Hence, it can be decomposed into two time series $X T$ and $Z T$. Initially, the trajectories are smoothed to remove noise. Then, each single smoothed trajectory $T_{x z}$ is partitioned into $N$ smaller subtrajectories $\left(S_{x z}=\left\{s_{i}\right\}_{i=1 \ldots N}\right)$. This partitioning allows us to search for work cycles that correspond to smaller segments of each trajectory $T_{x z}$. The partitioning is time-based. A representative speed value $\left(\left\|\vec{v}_{i}\right\|_{x z}\right)$ is then calculated for each subtrajectory $s_{i}$. Then, the speed values $\left(\left\|\vec{v}_{x z}\right\|=\left\|\vec{v}_{i}\right\|_{x z i=1 \ldots N}\right)$ of subtrajectories $S_{x z}$ are clustered in two groups to classify the subtrajectories $\left\{s_{i}\right\}_{i=1 \ldots N}$ as either 'stop' or 'move' semantic events. Finally, the classified subtrajectories $\left\{s_{i}\right\}_{i=1 \ldots N}$ are clustered into work cycles $\left(\left\{c_{i}\right\}_{i=1 \ldots K}\right)$ based on semantic criteria. The time a worker spends on a task (labour input) is equal to the total duration of these cycles.

The method presented in this paper makes four basic assumptions. The first and main assumption is that all construction-related tasks fit the same pattern. This pattern dictates that if a worker's 'move' is followed sequentially by one 'stop' and a second 'move', then these three semantic events define a work cycle. This assumption is based on the fact that workers 'stop' to perform a subtask and they 'move' to start another. The semantic 'move' event depicts the transition between subtasks, whereas the semantic 'stop' event depicts the actual execution of a subtask. Hence, the duration of a work cycle is equal to the duration of the semantic 'stop' event. Such work cycles depict the direct work that is currently used during activity analysis for workforce assessment. Direct work is defined as either 'the amount of time spent actively completing units output that contribute to the completion of a project' or 'the act of either exerting physical effort to perform an activity or of physically assisting in these activities'. Sequentially, the duration of all work cycles is equal to the labour input of a worker. Therefore, the labour input can be extracted by detecting these work cycles. The second assumption is that workers still have a range of movements while at a 'stop' that allows them to perform their tasks. This includes torso movement (i.e. forward bending, backward bending, sideways bending and rotation, knee bending) and sideway steps. The third assumption is that the speed generated $\left\|\vec{v}_{i}\right\|_{x z i=1 \ldots N}$ from the movements while at 'stop' is smaller than the average walking speed, which is equal to $1.4 \mathrm{~m} / \mathrm{s}$ (Boonstra et al., 1993). This assumption is illustrated in Figure 2. The fourth assumption is that the triangulation and calibration errors, which propagate as noise in the calculation of the computer vision-based 3D trajectories, have the same effect on all data sets regardless of the experimental settings - that is, the distance of cameras from workers, size of calibration board and indoors/outdoors monitoring.

\subsection{D trajectory calculation}

This paper combines two previously developed methods to calculate the 3D trajectories of workers. Both of these methods are designed for construction workers. The first is a computer vision-based method that tracks workers across subsequent frames of the same camera (Konstantinou et al., 2019). This method is 
Monitoring construction labour productivity by way of a smart

technology approach

Konstantinou and Brilakis

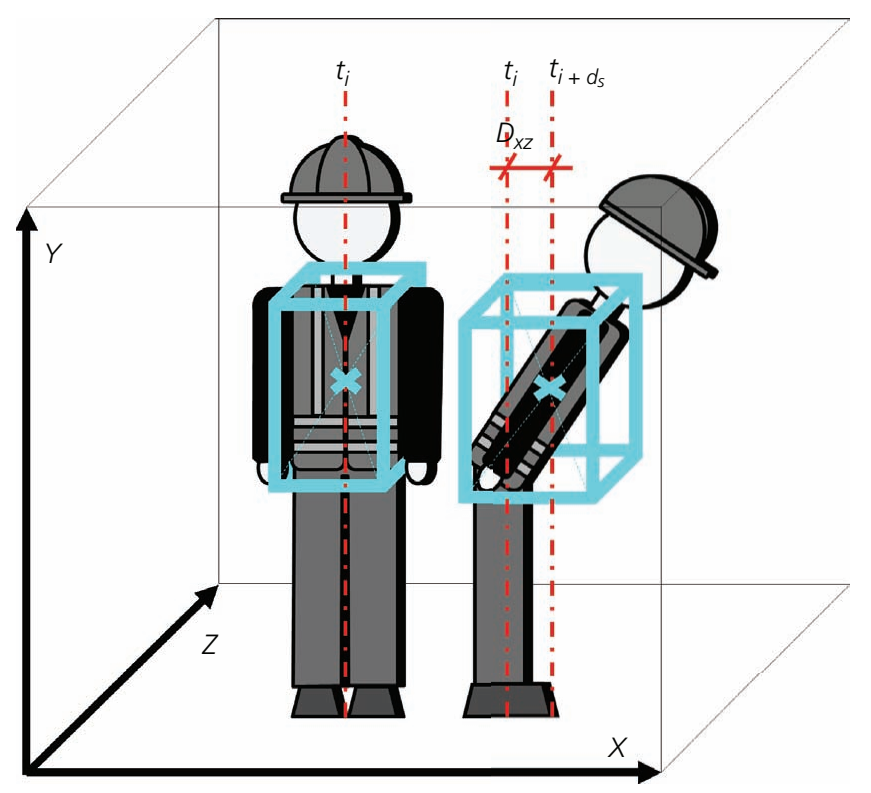

Figure 2. Forward bending while at 'stop' event

manually initiated and uses colour features to track multiple workers under various challenges such as illumination, posture, and scale variations, abrupt movements and occlusions. It returns the two-dimensional (2D) trajectories of the tracked entities. The second is a computer vision-based method that matches the $2 \mathrm{D}$ trajectories that belong to the same across multiple camera views using unique appearance features such as motion, geometric restrictions and colour features (Konstantinou and Brilakis, 2018b). The matched 2D trajectories are then triangulated (Hartley, 1997) to extract workers' 3D trajectories.

\subsection{Work cycle detection}

This section proposes a trajectory-based approach for detecting work cycles. The main hypothesis of this paper is that the labour productivity of construction workers can be monitored through their trajectory data.

\subsubsection{Noise filtering}

This section proposes a method to remove noise (smoothing) from the computer vision-based extracted 3D trajectories. Existing smoothing methods such as moving averages are robust for removing random noise from time series. They replace every data point of a time series by averaging $k$ previous points. These methods either treat all data points with the same significance or consider that the most recent ones are more important. The moving average methods are

simple moving average (SMA):

1.

$$
\widehat{x}_{t}=\frac{1}{k} \sum_{i=0}^{k-1} x_{t-i}, \text { for } t=k+1, \ldots, n
$$

weighted moving average (WMA):

$$
\widehat{x}_{t}=\frac{1}{\sum_{i=0}^{k-1} w_{i}} \sum_{i=0}^{k-1} w_{i} x_{t-i}, \text { for } t=k+1, \ldots, n
$$

exponential moving average (EMA):

$$
\begin{aligned}
\widehat{x}_{t} & =\lambda x_{t-1}+(1-\lambda) \widehat{x}_{t-1} \\
& =\sum_{i=0}^{k-1} \lambda(1-\lambda)^{i} x_{t-k}+(1-\lambda)^{t} \widehat{x}_{0}, \text { for } 0<\lambda \leq 1
\end{aligned}
$$

where weights $w_{i}$ and constant $\lambda$ of WMA and EMA, respectively, are chosen either based on the experience of the user and the nature of the data or through trial and error processes (Gor and Man, 2009). All moving average methods are experimentally evaluated in the following section. The limitation of such smoothing methods is that they also discard non-noise peak values if a large smoothing step $k$ is chosen (Guiñón et al., 2007). This section presents a two-step method that searches for the optimum smoothing step $k$ to alleviate this issue. It exploits the fact that all three time series $(X T, Y T$ and $Z T)$ of a worker who remains still are straight lines of zero degrees inclination. Firstly, all three time series are smoothed by a randomly selected initialisation step $\left(k_{i}\right)$. Secondly, simple linear regression fits a line $f_{t}=a x_{t}+b$ to every smoothed time series that minimises the sum of residuals $e_{t}=x_{t}-f_{t}$ (see Figure 3). Smoothing and fitting are repeatedly performed for all three time series. During this

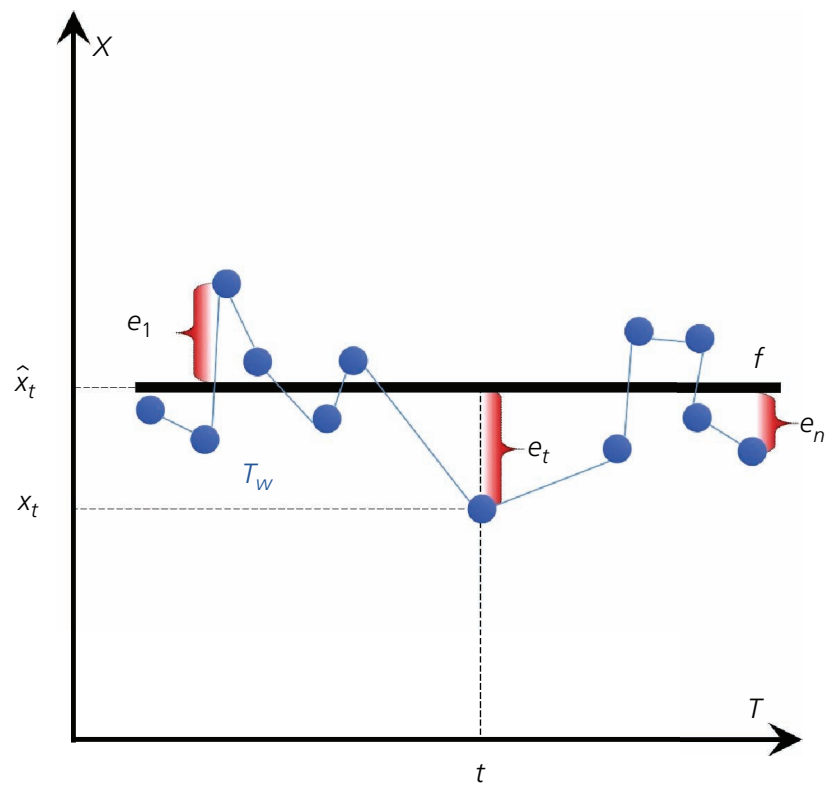

Figure 3. Fitting a line to a time series 
Smart Infrastructure and Construction Volume 172 Issue 2 searching, the smoothing step $k$ is increased successively. Then the proposed smoothing method searches for the smoothing step $k$ with the minimum inclination $\left(a_{x t_{k_{i}}}, a_{y t_{k_{i}}}, a_{z t_{k_{i}}}\right)$, for each of the three time series $\left(k_{x t}, k_{y t}, k_{z t}\right)$.

\subsubsection{Trajectory segmentation}

The partitioning of trajectories allows the detection of work cycles that are hidden in small segments of single trajectories. The proposed method partitions the $3 \mathrm{D}$ trajectories based on a time interval $\left(d_{s}\right)$ that aligns with the third assumption of this paper. Therefore, the time interval $d_{s}$ is set equal to $1 \mathrm{~s}$. Every trajectory $T_{x z}$ is partitioned into $N$ subtrajectories $S_{x z}=s_{i i=1 \ldots N}$ of an equal duration $d_{s}$. Each subtrajectory $s_{i}$ is then described by a representative speed value $\left\|\vec{v}_{i}\right\|_{x z}$ that depicts the motion of a worker along the floor plane and is expressed as follows:

4. $\left\|\vec{v}_{i}\right\|_{x z}=\sqrt{\left(\frac{x_{t}-x_{t-d_{s}}}{d_{s}}\right)^{2}+\left(\frac{z_{t}-z_{t-d_{s}}}{d_{s}}\right)^{2}}$

\subsubsection{Centroid clustering}

This section classifies the subtrajectories $\left\{s_{i}\right\}_{i=1 \ldots N}$ of workers as either 'stop' or 'move' semantic events by clustering their speed values $\left\|\vec{v}_{i}\right\|_{x z i=1 \ldots N}$. A distance-based clustering method is used to achieve this. The total number of clusters $(n)$ represents the 'stop' and 'move' events. Hence, it is set equal to 2. The subtrajectories $\left\{s_{i}\right\}_{i=1 \ldots N}$ that belong to the cluster with the smallest speed values $\left\|\vec{v}_{i}\right\|_{x z i=1 \ldots N}$ are labelled as 'stop', while the subtrajectories that belong to the cluster with the largest speed values $\left\|\vec{v}_{i}\right\|_{x z i=1 \ldots N}$ are labelled as 'move'. Therefore, each subtrajectory $s_{i}$ is classified as follows:

5. $\quad s_{i}=\left\{\begin{array}{l}\text { 'stop', if : }\left\|\vec{v}_{i}\right\|_{x z} \in \text { centroid }\left\|\vec{v}_{i}\right\|_{x z \min } \\ \text { 'move', if }:\left\|\vec{v}_{i}\right\|_{x z} \in \text { centroid } \vec{v}_{i} \|_{x z \max }\end{array}\right.$

where centroid $\vec{v}_{i} \|_{x z_{\min }}$ and centroid $\vec{v}_{i} \|_{x z_{\max }}$ are the minimum and maximum values, respectively, of the clusters' centroids. The $K$-means algorithm is used for clustering the speed values $\left\|\vec{v}_{i}\right\|_{x z i=1 \ldots N}$. Such clustering approach is selected as it converges successfully to the global optimum for well-separated clusters.

\subsubsection{Semantic clustering}

This section presents how all classified subtrajectories $\left\{s_{i}\right\}_{i=1 \ldots N}$ are further clustered into work cycles. Initially, all subtrajectories $\left\{s_{i}\right\}_{i=1 \ldots N}$ that are sequentially classified as either part of 'stop' or 'move' events are grouped into semantic events of larger duration (see Figure 4). Then, the proposed clustering method relies on the main assumption of this paper to detect the work cycles $\left\{c_{i}\right\}_{i=1 \ldots K}$. This assumption dictates that if one 'move' event is followed by one 'stop' and one 'move' event sequentially, then all

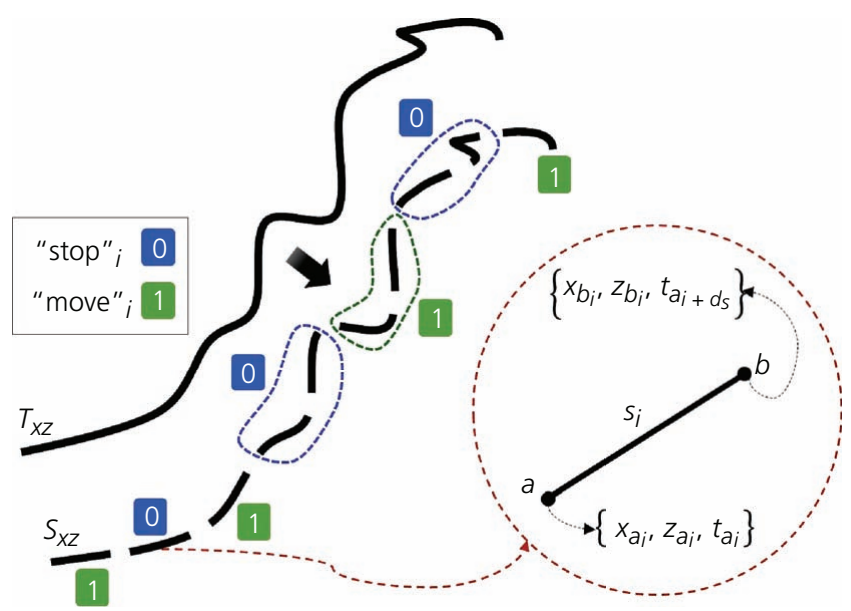

Figure 4. Classification of subtrajectories $\left\{s_{i}\right\}_{i=1 \ldots N}$ into semantic events

subtrajectories $\left\{s_{i}\right\}_{i=1 \ldots N}$ that belong to these three sequential semantic events depict a work cycle $c_{i}$. This is expressed as follows:

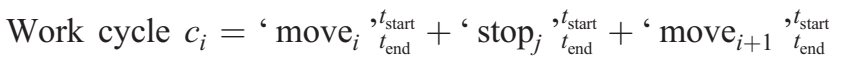
6.

The starting $t_{\text {start }}$ and ending $t_{\text {end }}$ times of a semantic event are equal to the starting and ending times of its first and last subtrajectories, respectively. Figure 5 illustrates how the semantic events are clustered into work cycles $\left\{c_{i}\right\}_{i=1 \ldots K}$. The duration of each work cycle $c_{i}\left(d_{c_{i}}\right)$ is equal to the duration of the 'stop' event ( $d_{\text {'stop'). }}$,

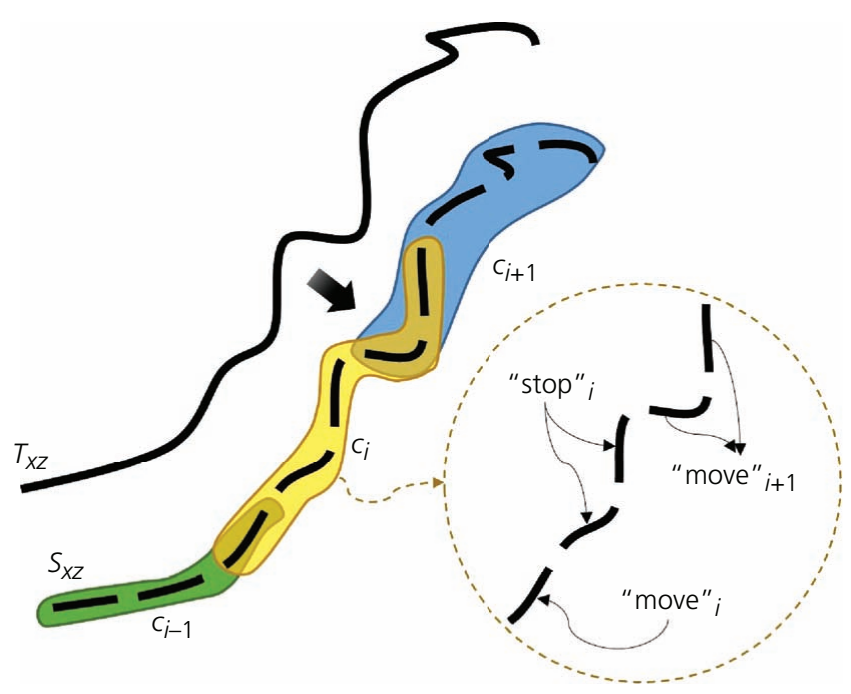

Figure 5. Semantic clustering of subtrajectories $\left\{s_{i}\right\}_{i=1 \ldots N}$ into work cycles $\left\{c_{i}\right\}_{i=1} \ldots K$ 
Smart Infrastructure and Construction Volume 172 Issue 2
Monitoring construction labour productivity by way of a smart technology approach

Konstantinou and Brilakis

\section{Implementation and results}

This section presents the performance of the overall proposed method in terms of $(a)$ detecting work cycles and $(b)$ determining the labour input of construction workers. A C\# implementation in Microsoft Visual Studio.Net framework, running in a Windows 8.1 operating system, is developed to achieve this (see Figure 6). More specifically, this software development $(a)$ processes multiple videos at the same time, $(b)$ tracks multiple workers across subsequent frames of the same camera, $(c)$ performs camera calibration, $(d)$ matches the same workers across multiple camera views, $(e)$ calculates the 3D trajectories of workers across the jobsite, $(f)$ displays the spaghetti diagrams that depict workers' motion on the floor plane and $(g)$ calculates the work cycles of multiple workers. A desktop PC with the following specs is used: Intel core i7 CPU, 4.0 GHz and 32 GB RAM. consists of two workers who perform an electrical task (data set electrical). The total duration of data set steel fixing and electrical are 35 and $39 \mathrm{~min}$, respectively.

The data were collected with two GoPro cameras, black edition 4 with $1920 \times 1080$ frame size and 30 frames per second (fps) frame rate. The cameras were placed on a tripod, as illustrated in Figure $8(\mathrm{c})$. This paper used a $13 \times 9$ chessboard with a $60-\mathrm{mm}$ square size as a calibration pattern (see Figure 8(a) and 8(b)) for calibrating the cameras (Hartley, 1997; Zhang, 2000). The quality of the proposed method's evaluation process depends on the sample size to be tested. This evaluation section defines the minimum number of required samples $n$ through the following equation (Eng, 2003):

The performance of the proposed method is evaluated with two data sets (see Figure 7). The first captures one worker while performing a steel fixing task (data set steel fixing). The second

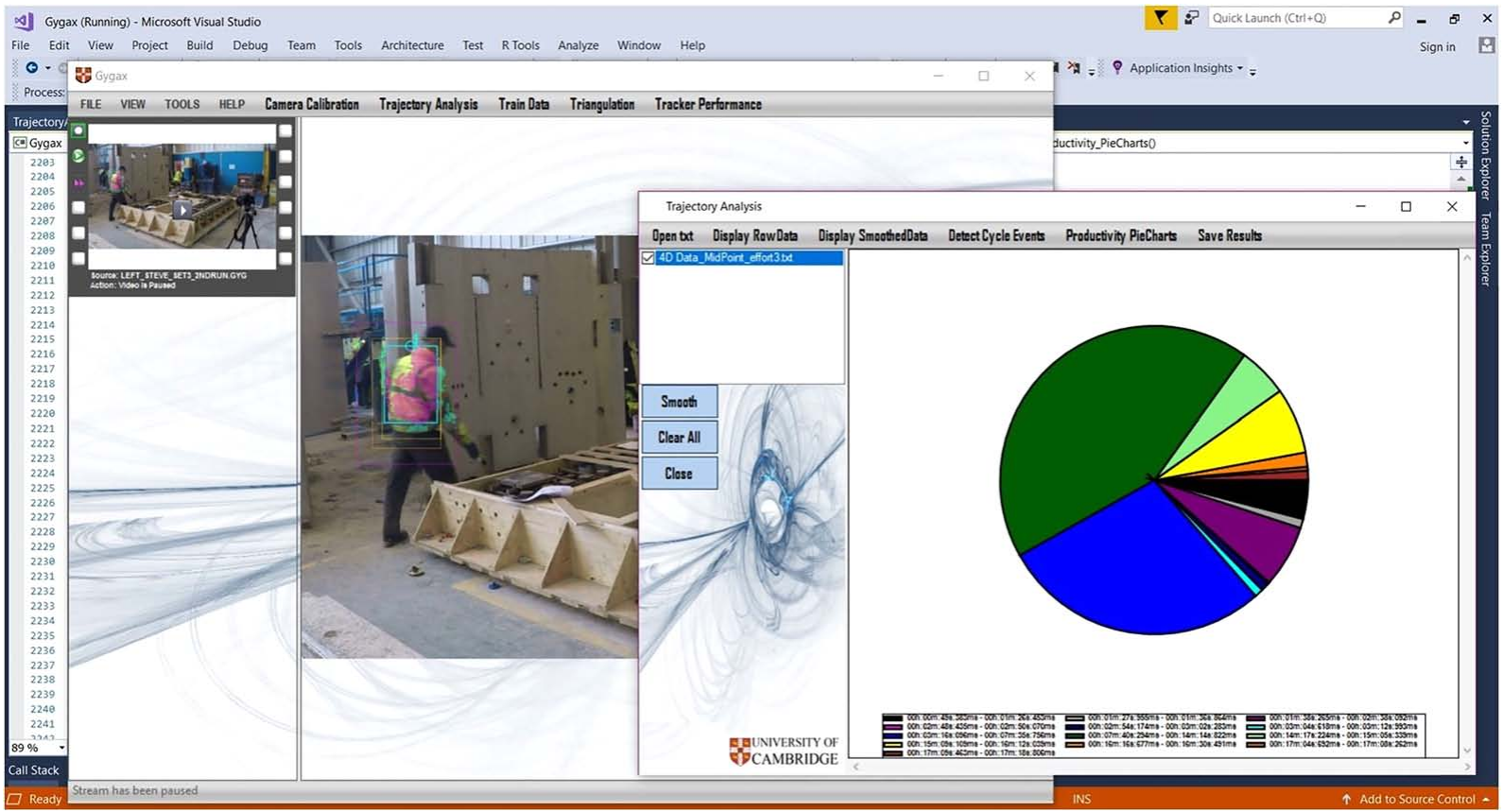

Figure 6. Microsoft Visual Studio.Net framework for monitoring labour productivity
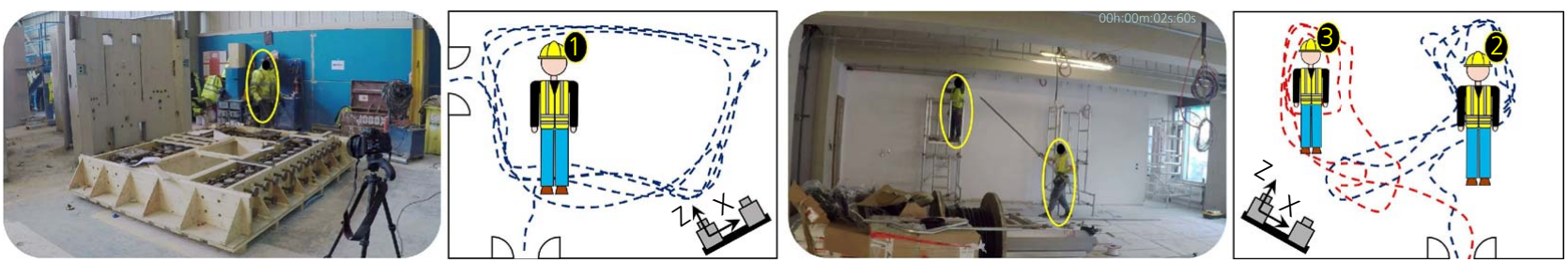

Figure 7. Tested data sets (from left to right: data set steel fixing, data set electrical) 


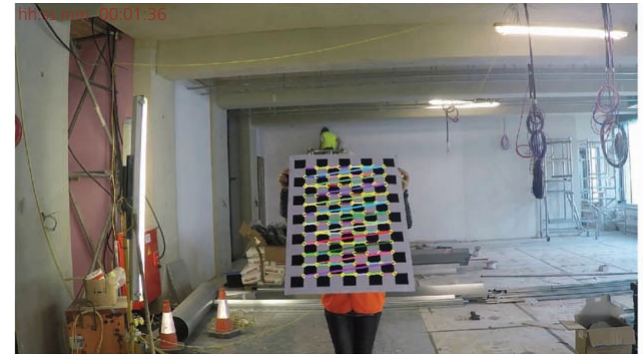

(a)

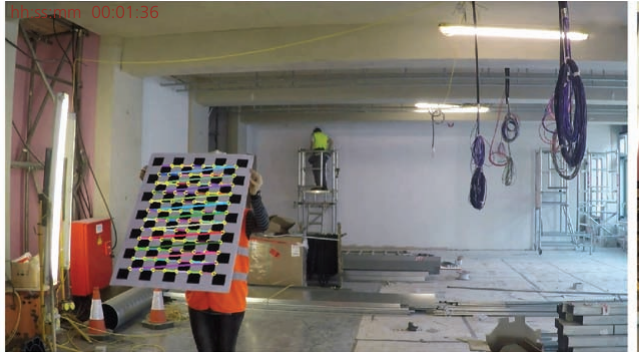

(b)

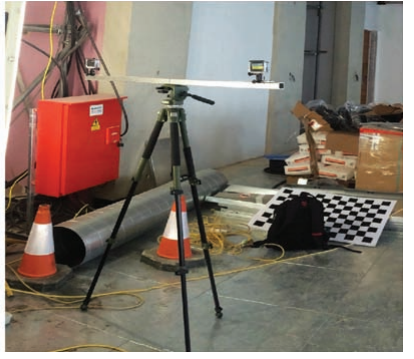

(c)

Figure 8. Chessboard detection in (a) left and (b) right camera view and (c) stereo camera setup

where $Z_{\text {crit }}$ is the confidence level of $90 \%\left(Z_{\text {crit }}=1.645\right), D$ is the limit of error equal to $\pm 10 \%$ and $p$ is the proportion of each sample with a specific characteristic and is equal to $32 \%$ for both data sets. This characteristic refers to work cycles that consist of semantic events with a duration $\left(t_{\text {start }}-t_{\text {end }}\right)$ smaller than $1 \mathrm{~s}$ (see Equation 6). Such work cycles cannot be detected. This is due to the time interval $\left(d_{s}=1 \mathrm{~s}\right)$ used for segmenting the trajectories. The minimum required sample $n$ is equal to 59. Precision, recall and accuracy metrics are used for the evaluation of the proposed method. Precision is the fraction of the total number of correctly detected work cycles (TP, true positive) over the total number of incorrectly and correctly detected work cycles (true positive (TP) + false positive (FP)). Recall depicts the detection completion level and is equal to the total number of correctly detected work cycles (TP) divided by the total number of correctly detected and incorrectly not detected work cycles (TP + false negative $(\mathrm{FN})$ ). Lastly, accuracy is defined by the number of correctly detected work cycles (TP) and the number of work cycles that were correctly not detected (true negative (TN)) over the total sum of work cycles. The work cycles are manually measured for the evaluation of the proposed method.

\subsection{Definition of smoothing parameters}

This section determines experimentally the smoothing step $k$ to remove noise from workers' $3 \mathrm{D}$ trajectories. The proposed noise filtering method returns a smoothing step for each of the three time series $\left(k_{x t}, k_{y t}, k_{z t}\right)$. This section uses the 3D trajectories of a worker who remains still to achieve this (see Figure 9). Given that the worker in this example does not move, the $X T, Y T$ and $Z T$ time series should display zero motion. The performance of each smoothing method is illustrated in Table 1 and is calculated based on the following distance errors:

8. $x_{\text {error }}=\left|x_{\max }-x_{\min }\right|$

9. $y_{\text {error }}=\left|y_{\max }-y_{\min }\right|$

10. $z_{\text {error }}=\left|z_{\max }-z_{\min }\right|$

EMA is evaluated for the entire range of $\lambda$ values $(0<\lambda \leq)$. It appears that WMA performs the best compared with SMA and EMA as it returns the smallest errors. The second best option is EMA for $\lambda=0.1$. In particular, the proposed filtering method with WMA features a maximum error equal to $3 \mathrm{~cm}$ error along the $X$-axis, $0.3 \mathrm{~cm}$ along the $Y$-axis and $1.2 \mathrm{~cm}$ along the $Z$-axis. Therefore, the smoothing steps that are selected for the $X, Y$ and $Z$ time series are 772,1187 and 1155 , respectively. Figure 10 illustrates the performance of the proposed filtering method with WMA. As it appears in Figure 10(a) and 10(b), the ZT time series is the one with the most noise. Due to this, the worker in the example of Figure 9 wrongly appears as if he has moved $0.45 \mathrm{~m}$ along the $X$-axis and almost $1 \mathrm{~m}$ along the $Z$-axis. The almost perfectly linear (i.e. no motion) time series $(X T, Y T$ and $Z T)$ in Figure 10(c) show that the proposed method removes noise successfully.

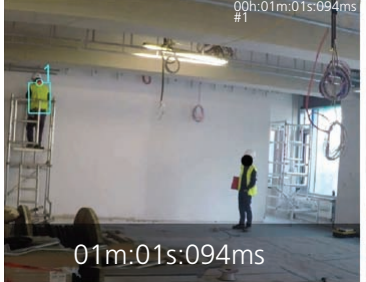

(a)

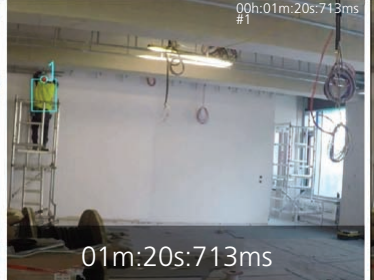

(b)

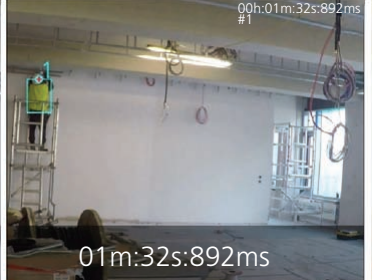

(c)

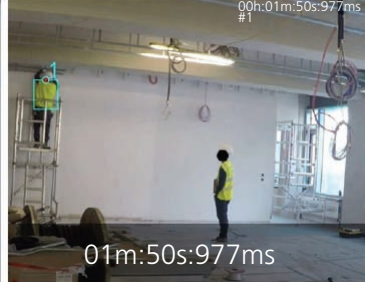

(d)

Figure 9. Screenshots of a tracked worker (in rectangle) who remains still 
Table 1. Smoothing parameters of the proposed noise filtering method with SMA, EMA and WMA

\begin{tabular}{|c|c|c|c|c|c|c|c|c|c|c|c|c|}
\hline & \multirow{2}{*}{ SMA } & \multirow{2}{*}{ WMA } & \multicolumn{10}{|c|}{ EMA } \\
\hline & & & 1.0 & 0.9 & 0.8 & 0.7 & 0.6 & 0.5 & 0.4 & 0.3 & 0.2 & 0.1 \\
\hline$k_{x t}$ & 27 & 772 & 287 & 288 & 288 & 288 & 289 & 289 & 292 & 292 & 290 & 295 \\
\hline$k_{y t}$ & 1208 & 1187 & 1355 & 701 & 1405 & 702 & 702 & 702 & 702 & 706 & 709 & 719 \\
\hline$k_{z t}$ & 0 & 1155 & 1203 & 817 & 817 & 599 & 1241 & 878 & 1206 & 819 & 820 & 822 \\
\hline$x_{\text {error }}$ & 3.64 & 0.03 & 0.16 & 0.16 & 0.16 & 0.16 & 0.16 & 0.16 & 0.16 & 0.15 & 0.15 & 0.14 \\
\hline yerror & 2.51 & 0.003 & 0.06 & 0.04 & 0.04 & 0.13 & 0.12 & 0.12 & 0.11 & 0.10 & 0.09 & 0.07 \\
\hline$Z_{\text {error }}$ & 1.30 & 0.012 & 0.99 & 1.16 & 1.10 & 1.04 & 0.79 & 0.93 & 0.66 & 0.76 & 0.66 & 0.51 \\
\hline
\end{tabular}

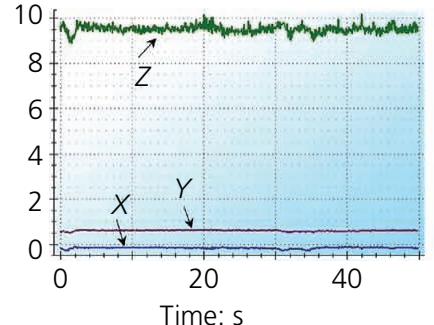

(a)

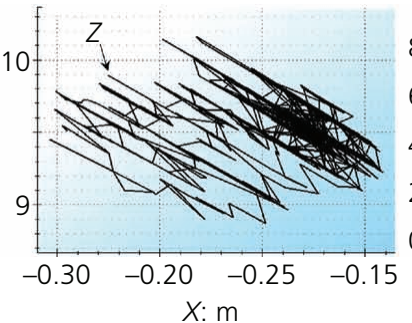

(b)

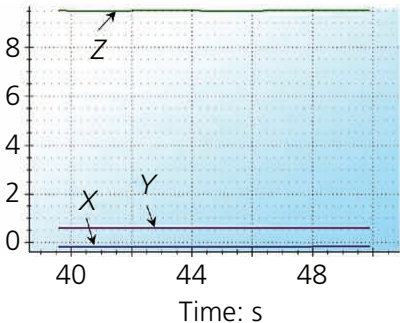

(c)

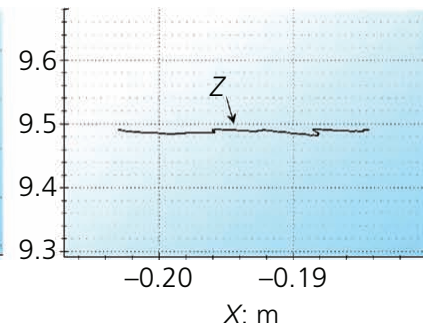

(d)

Figure 10. Performance of the proposed noise filtering method on a non-moving worker (see Figure 9). (a) Unsmoothed 3D time series and (b) $T_{x z}$. (c) Smoothed 3D time series and (d) $T_{x z}$ (see Figure 9)

\subsection{Evaluation of work cycles' detection}

This section evaluates the performance of the method presented in this paper in terms of translating the trajectory data into labour input through the detection of work cycles. In this section, the pie charts in Figure 11 and Figure 13 show $(a)$ the durations of the manually collected ground truth (GT) work cycles that correspond to TP results (the ones with semantic events smaller than $d_{s}$ are not displayed for figure legibility reasons), $(b)$ the detected work cycles using the method proposed in this paper and (c) the trajectories of workers $T_{x z}$ along the floor plane over time. The
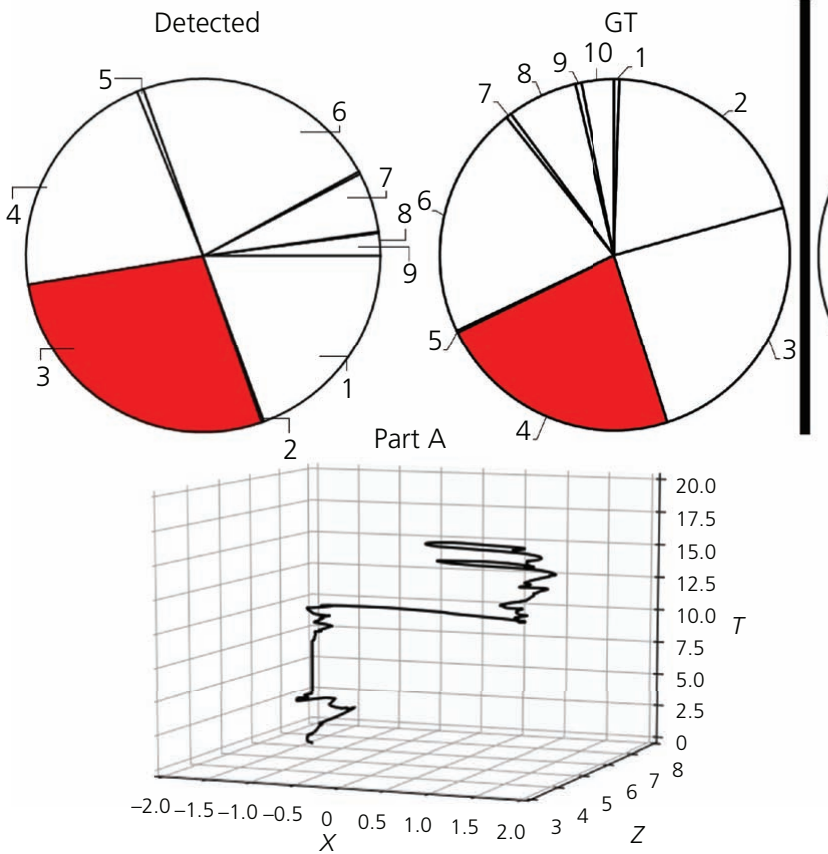

Detected
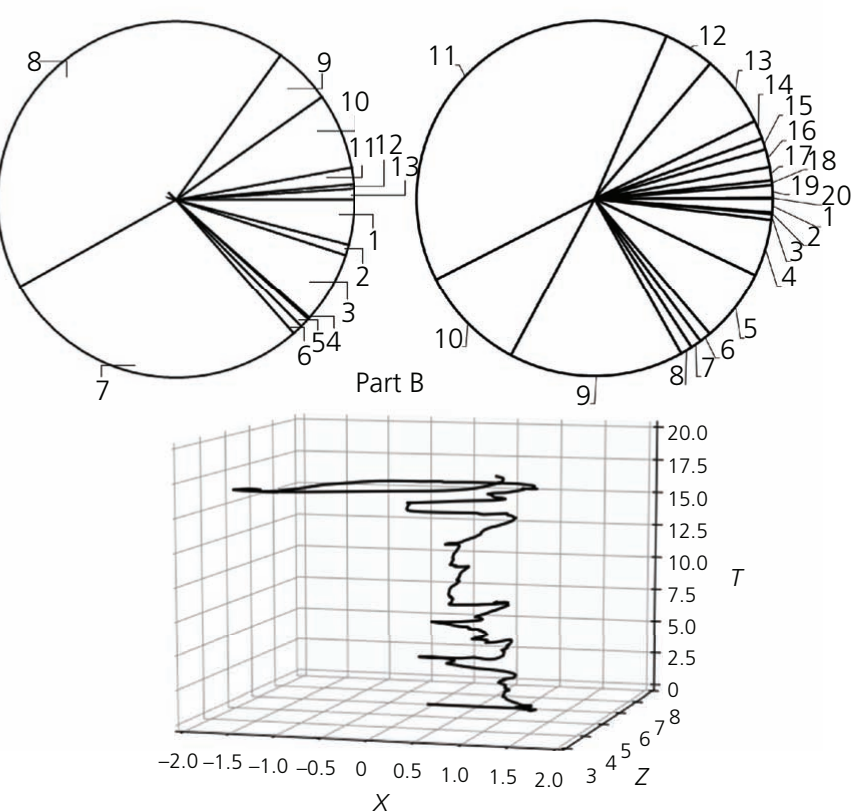

Figure 11. Detected work cycles of worker '1' from data set steel fixing 
dark shaded work cycles in these figures depict absenteeism time - that is, the time workers leave the field of view of cameras.

Table 2-Table 4 display in detail the GT of all work cycles, including the ones with a time interval smaller than $1 \mathrm{~s}$.

\subsubsection{Steel fixing task}

The first data set consists of two recordings (parts A and B) with a duration of approximately $17 \mathrm{~min}$ each. Both recordings are collected the same day. Figure 11 displays the GT and the detected work cycles of worker ' 1 ' for both parts A and B. In total, worker ' 1 ' performed 43 work cycles. These cycles depict the following subtasks: $(a)$ fixing steel re-bars, $(b)$ picking re-bars or equipment and $(c)$ reading drawings. The proposed method detects $8 \mathrm{TP}, 6 \mathrm{TN}, 1 \mathrm{FP}$ and $0 \mathrm{FN}$ in part A and $12 \mathrm{TP}, 13 \mathrm{TN}, 0$ $\mathrm{FP}$ and $4 \mathrm{FN}$ in part $\mathrm{B}$. In total, the $14 \mathrm{TN}$ correspond to GT work cycles with a duration smaller than $d_{s}=1 \mathrm{~s}$. For example,

Table 2 shows that the TN $(* 2)$ in part A occurs as the time interval between the start $\left(t_{\text {start }}=11 \mathrm{~m}: 37 \mathrm{~s}: 097 \mathrm{~ms}\right)$ and the end $\left(t_{\text {end }}=11 \mathrm{~m}: 38 \mathrm{~s}: 064 \mathrm{~ms}\right)$ of the 'move int $_{i+1} t_{\text {sart }}$ event of this work cycle (see Equation 6) is less than $d_{s}=1 \mathrm{~s}$. The other $4 \mathrm{TN}$ work

Table 2. GT of semantic 'stops' of worker ' 1 ' (data set steel/parts A and B)

\begin{tabular}{llc}
\hline Part A (GT)/start-end & Part B (GT)/start-end \\
\hline$\left[{ }^{*} 1\right] 00: 00: 033-00: 05: 105$ & {$\left[{ }^{*} 1\right] 00: 01: 701-00: 14: 114$} & {$[9] 03: 12: 892-05: 53: 687$} \\
{$[1]$ 00:00:033-00:05:105 } & {$\left[{ }^{*} 2\right] 00: 19: 953-00: 21: 287$} & {$[10] 05: 55: 021-07: 33: 353$} \\
{$[2] 00: 12: 645-03: 37: 884$} & {$\left[{ }^{*}\right] 00: 25: 258-00: 30: 697$} & {$\left[{ }^{*} 9\right] 07: 37: 657-12: 30: 050$} \\
{$[3] 03: 39: 153-07: 48: 035^{* *}$} & {$\left[{ }^{*} 4\right] 00: 33: 867-00: 34: 768$} & {$[11] 07: 37: 657-14: 14: 087$} \\
{$\left[{ }^{*} 2\right] 07: 50: 036-11: 37: 097$} & {$\left[{ }^{*}\right] 00: 37: 771-01: 25: 018$} & {$[12] 14: 15: 388-15: 02: 268$} \\
{$\left[{ }^{*} 3\right] 11: 38: 064-11: 39: 532$} & {$[1] 00: 01: 701-00: 14: 114$} & {$[13] 15: 04: 204-16: 10: 503$} \\
{$[4] 07: 50: 036-11: 41: 067$} & {$[2] 00: 19: 953-00: 21: 287$} & {$\left[{ }^{*} 10\right] 16: 12: 038-02: 17: 644$} \\
{$[5] 11: 42: 268-11: 44: 070$} & {$[3] 00: 25: 258-00: 30: 697$} & {$[14] 16: 18: 645-16: 25: 185$} \\
{$\left[{ }^{*} 4\right] 11: 52: 245-15: 30: 096$} & {$[4] 00: 33: 867-01: 26: 386$} & {$[15] 16: 31: 958-16: 29: 122$} \\
{$\left[{ }^{*}\right] 15: 30: 997-15: 30: 196$} & {$\left[{ }^{*} 6\right] 01: 28: 188-02: 33: 053$} & {$[16] 16: 46: 139-17: 02: 422$} \\
{$[6] 11: 52: 245-15: 30: 196$} & {$[5] 01: 28: 188-02: 36: 322$} & {$\left[{ }^{*} 12\right] 17: 05: 658-17: 08: 028$} \\
{$[7] 15: 35: 101-15: 40: 273$} & {$\left[{ }^{*} 7\right] 02: 40: 293-02: 47: 801$} & {$[17] 17: 05: 658-17: 17: 904$} \\
{$[8] 15: 43: 610-16: 49: 609$} & {$[6] 02: 40: 293-02: 50: 970$} & {$\left[{ }^{*} 13\right] 17: 23: 943-17: 26: 579$} \\
{$\left[{ }^{*} 6\right] 16: 57: 317-16: 58: 618$} & {$[7] 02: 52: 205-03: 02: 115$} & {$[18] 17: 23: 943-17: 28: 381$} \\
{$[9] 16: 57: 317-17: 02: 989$} & {$[8] 03: 01: 014-03: 11: 858$} & {$[19] 17: 30: 517-17: 41: 728$} \\
{$[10] 17: 11: 131-17: 42: 962$} & {$\left[{ }^{*} 8\right] 03: 12: 892-05: 42: 442$} & {$[20] 17: 41: 728-17: 42: 929$}
\end{tabular}

[*] depicts TN, ${ }^{* *}$ depicts absenteeism, time format (m:s:ms).

Table 3. GT of semantic 'stops' of workers ' 2 ' and ' 3 ' (data set electrical/part A)

\begin{tabular}{|clll|}
\hline Part A '2' (GT)/start-end & & Part A '3' (GT)/start-end & \\
\hline$\left[{ }^{*} 1\right] 00: 00: 033-03: 52: 765$ & {$[1] 00: 00: 033-05: 20: 586$} & {$[7] 07: 33: 920-08: 17: 263^{* *}$} & {$[9] 08: 25: 872-08: 50: 536$} \\
{$[1] 00: 00: 033-07: 50: 536$} & {$[2] 05: 23: 823-05: 31: 264^{* *}$} & {$\left[{ }^{*}\right]$ 08:18:698-08:21:501 } & {$[10] 08: 36: 749-08: 50: 536$} \\
{$[2] 07: 40: 640-08: 11: 190^{* *}$} & {$\left[{ }^{*} 1\right]$ 05:33:566-05:39:839 } & {$\left[{ }^{*} 4\right]$ 08:25:872-08:33:145 } & {$[11] 08: 41: 988-08: 50: 536$} \\
{$\left[2^{*}\right] 08: 15: 561-08: 38: 551$} & {$\left[{ }^{*}\right]$ 05:41:908-05:43:876 } & {$\left[{ }^{*} 5\right]$ 08:36:749-08:39:652 } & {$[12] 08: 45: 992-09: 50: 536$} \\
{$[4] 08: 40: 219-11: 49: 608$} & {$[3] 05: 33: 566-05: 50: 536$} & {$\left[{ }^{*} 6\right]$ 08:41:988-08:43:356 } & {$[13] 09: 08: 180-09: 50: 536$} \\
- & {$[4] 05: 41: 908-05: 50: 536$} & {$\left[{ }^{*} 7\right] 08: 54: 200-09: 04: 911$} & {$[14] 09: 30: 136-10: 50: 536$} \\
- & {$[5] 05: 46: 112-07: 50: 536$} & {$\left[{ }^{*} 8\right]$ 09:08:180-09:10:283} & {$[15] 10: 10: 309-11: 50: 536$} \\
- & {$[6] 07: 11: 063-07: 50: 536$} & {$[8] 08: 18: 698-08: 50: 536$} & -
\end{tabular}

[*] depicts TN, ${ }^{* *}$ depicts absenteeism, time format (m:s:ms).

Table 4. GT of semantic 'stops' of workers ' 2 ' and ' 3 ' (data set electrical/part B)

\begin{tabular}{|llll|}
\hline Part B '2' (GT) start-end & Part B '3' (GT) start-end & Part B '2' (GT) start-end & Part B '3' (GT) start-end \\
\hline$\left[{ }^{*} 1\right] 00: 00: 033-00: 03: 003$ & {$[7] 08: 25: 872-08: 33: 145$} & {$\left[{ }^{*} 1\right]$ 00:00:033-06:33:393 } & {$\left[{ }^{*} 4\right]$ 07:07:026-07:09:128 } \\
{$[1] 00: 00: 033-05: 20: 586$} & {$[8] 08: 36: 749-08: 39: 652$} & {$[1] 00: 00: 033-06: 37: 130$} & {$[4] 07: 07: 026-08: 00: 580$} \\
{$[2] 05: 33: 566-05: 39: 839$} & {$[9] 08: 41: 988-08: 43: 356$} & {$\left[{ }^{*}\right]$ 06:50:176-06:51:611 } & {$[* 5] 07: 11: 130-07: 16: 302$} \\
{$[3] 05: 41: 908-05: 43: 876$} & {$[10] 08: 45: 992-09: 04: 911$} & {$[* 3]$ 06:52:512-06:52:879 } & {$[5] 09: 26: 532-09: 28: 534 * *$} \\
{$[4] 05: 46: 112-07: 07: 593$} & {$[11] 09: 08: 180-09: 13: 286$} & {$[2] 06: 50: 176-06: 52: 879$} & {$[6] 09: 32: 204-11: 07: 166$} \\
{$[5] 07: 11: 063-07: 31: 017$} & {$[12] 09: 30: 136-10: 09: 208$} & {$[3] 07: 00: 887-07: 26: 112$} & - \\
{$[6] 08: 18: 698-08: 21: 501$} & {$[13] 10: 10: 309-11: 49: 608$} & - & -
\end{tabular}

$\left[{ }^{*}\right]$ depicts TN, ${ }^{* *}$ depicts absenteeism, time format (m:s:ms). 
cycles are caused by the way trajectories are smoothed. The maximum smoothing step $k$ of all three time series $\left(k_{y t}\right)$ is equal to $40 \mathrm{~s}$ if divided by the camera frame rate - that is, $\frac{k}{f p s}=\frac{1187}{30}$. Subsequently, all smoothed time series are $40 \mathrm{~s}$ shorter in length at the beginning compared with the unsmoothed time series. Hence, the work cycles TN $(* 1)$ of part A and TN $(* 1, * 2, * 3$, *4) of part B that fall within the initial $40 \mathrm{~s}$ cannot be detected. The FP and FN are due to the fluctuation of the implemented computer vision-based 2D tracking method. This fluctuation is illustrated in Figure 12. During these fluctuations, the tracker slides away from the target briefly.

\subsubsection{Electrical fixing task}

This data set consists of two recordings (parts A and B) that were all collected the same day. The duration of part A is 11 min and of part $\mathrm{C}$ is $12 \mathrm{~min}$. Figure 11 and Figure 13 illustrate the GT work cycles of worker ' 2 ' and worker ' 3 ' for parts $A$ and B, respectively. Overall, worker '2' performed seven work cycles, and worker ' 3 ' performed 24.

The proposed method detects $2 \mathrm{TP}, 1 \mathrm{TN}, 0 \mathrm{FP}$ and $0 \mathrm{FN}$ for worker ' 2 ' in part A and $2 \mathrm{TP}, 1 \mathrm{TN}, 4 \mathrm{FP}$ and $4 \mathrm{FN}$ in part $\mathrm{B}$. For worker ' 3 ', the proposed method returns $6 \mathrm{TP}, 8 \mathrm{TN}, 0 \mathrm{FP}$ and $0 \mathrm{FN}$ in part $\mathrm{A}$ and $5 \mathrm{TP}, 5 \mathrm{TN}, 3 \mathrm{FP}$ and $0 \mathrm{FN}$. The $15 \mathrm{TN}$ predictions for workers ' 2 ' and ' 3 ' in both parts $\mathrm{A}$ and $\mathrm{B}$ result either from work cycles with a time interval smaller than $d_{s}=1 \mathrm{~s}$ or due to the smoothing step applied similarly to the TN outcomes of worker ' 1 '. For example, in Table 4, the TN (*2) prediction of worker ' 3 ' in part B is due to the short time interval between the start $\left(t_{\text {start }}=06 \mathrm{~m}: 51 \mathrm{~s}: 611 \mathrm{~ms}\right)$ and the end $\left(t_{\text {end }}=\right.$ $06 \mathrm{~m}: 52 \mathrm{~s}: 512 \mathrm{~ms}$ ) of the ' $\mathrm{move}_{i+1}, t_{\text {sart }} t_{\text {end }}($ see Equation 6). The FP and $\mathrm{FN}$ are due to the distance error of the computer vision-based 2D tracking method, similar to that in data set steel fixing.

Table 5 summarises the performance of the method proposed in this paper in terms of detecting work cycles for the purpose of converting walking path trajectories into labour input. Overall, the proposed method features $81 \%$ precision, $90 \%$ recall and $85 \%$ accuracy. The small number of FP and FN results shows that the proposed method is not significantly affected by noise. The sum of the duration of both TP and FP work cycles of each worker depicts his/her labour input (see Table 6). On average, the proposed method returns an accuracy of $84 \%$ in terms of calculating the labour input.

\section{Conclusions}

It is reported that the construction sector has not improved labour productivity since 1960 . This is due to several factors that appear randomly on-site. Some of them are overtime, safety, resources, congestion, disruptions, site layout, skills, fatigue, absenteeism and unscheduled breaks. Considering that these factors are not periodic phenomena, significant amounts of time might be lost until they are redetected. Therefore, it is essential that labour productivity is monitored proactively. However, currently, this is not feasible as existing practices are extremely labour-intensive and time-consuming.

Previous studies focus on monitoring the labour input either by linking the location of workers to predefined work zones of specific management interest (e.g. excavation zone and brick laying zone) or by linking the activities of workers (e.g. bending, stretching, sound and strain) to specific tasks (e.g. nailing and brick laying). However, none of the existing studies have managed to monitor labour productivity for multiple construction workers at the same time, accurately, unobtrusively and time- and cost-efficiently.

This paper proposes a novel trajectory-based method to address this shortcoming. The novelty of the proposed method lies in trajectory clustering. This is achieved in two steps. Firstly, the proposed method performs a computer vision-based 3D tracking of construction workers. Secondly, the extracted 3D trajectories are segmented into subtrajectories, which are classified as either 'move' or 'stop' semantic events in order to be finally clustered

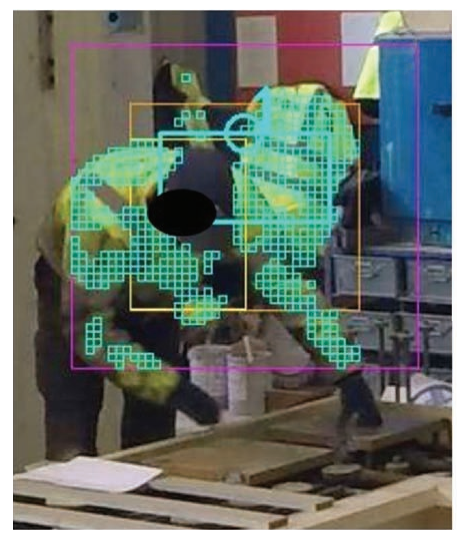

(a)

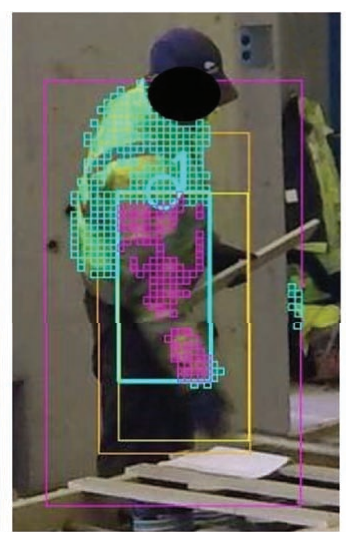

(b)

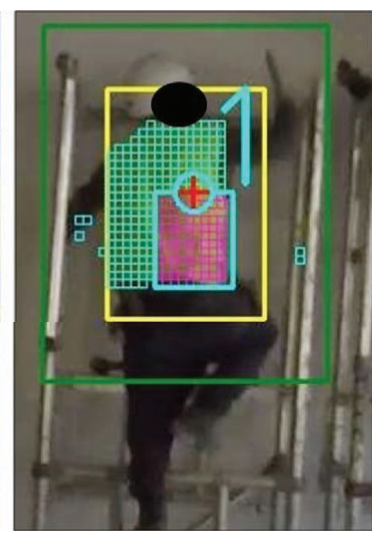

(c)

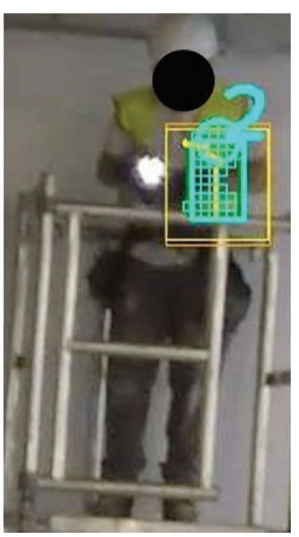

(d)

Figure 12. Fluctuation of the implemented computer vision-based 2D tracking method 

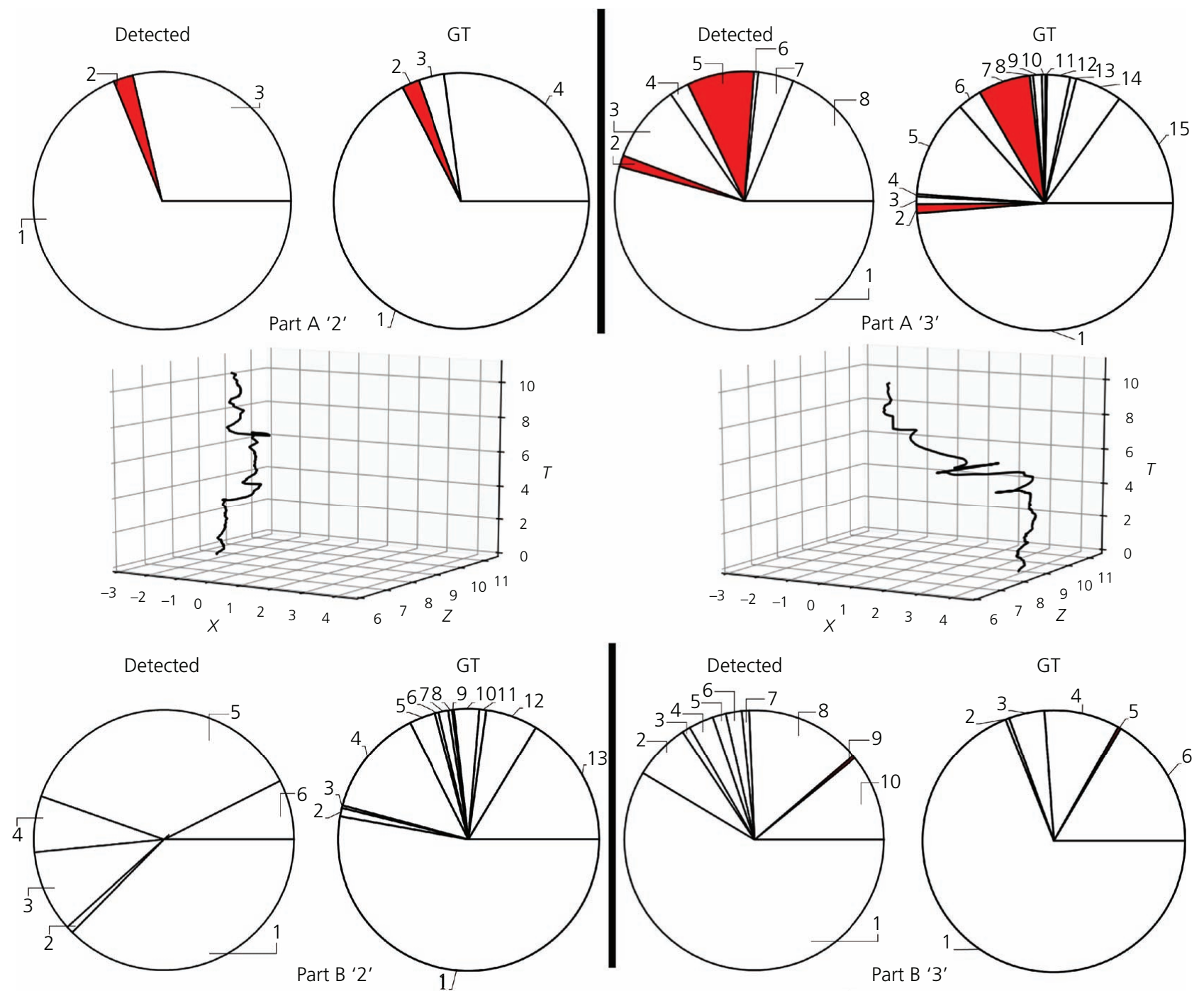

GT

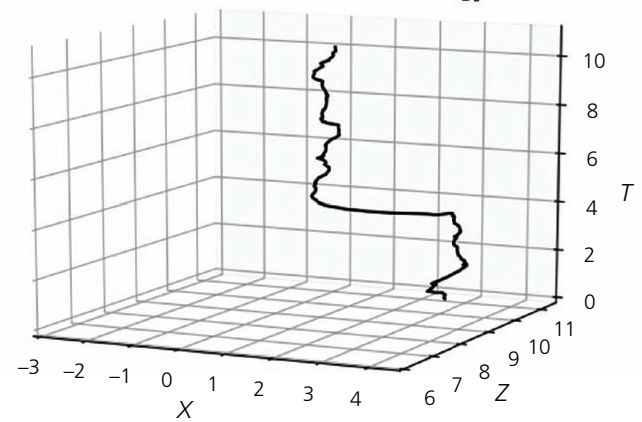

Part B '3'
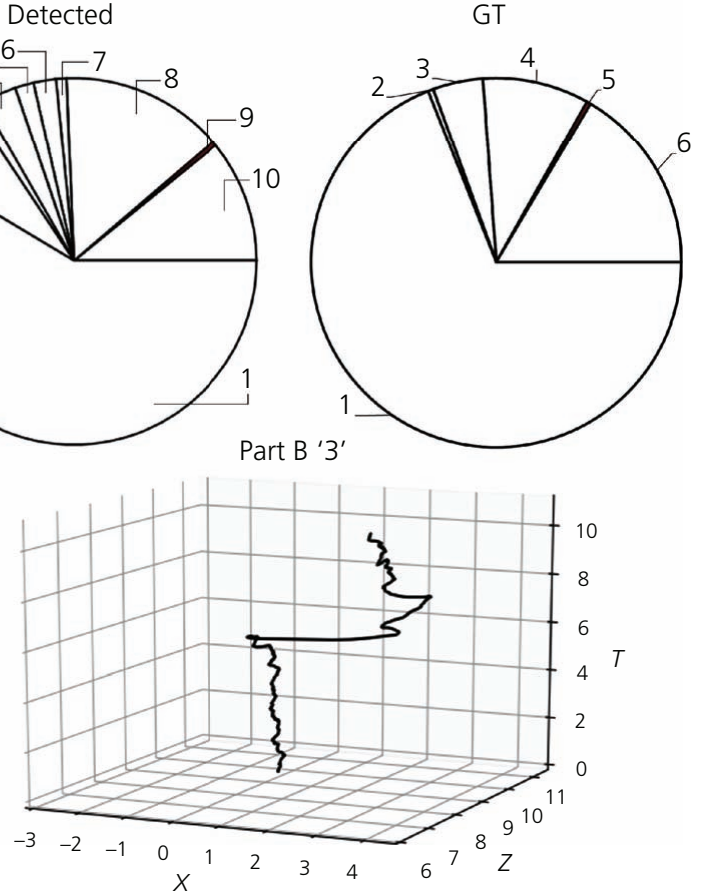

Figure 13. Detected work cycles of workers ' 2 ' and ' 3 ' for data set electrical fixing

into work cycles based on the main assumption of this paper that every work cycle is described by two semantic 'move' events and one semantic 'stop' event. The total duration of these work cycles is equal to a worker's labour input - that is, direct work.

The proposed productivity monitoring method is tested on a statistically sufficient sample size, which is collected at real construction jobsites depicting workers performing two different types of tasks: steel fixing and electrical. In particular, it features a precision of $81 \%$, a recall of $90 \%$ and an accuracy of $85 \%$ in terms of detecting work cycles and an average accuracy of $84 \%$ in terms of determining the total time (labour input/direct work) workers spend on construction-related tasks. The performance achieved shows that $(a)$ labour productivity of multiple workers 
Table 5. Confusion matrix of the proposed method in terms of detecting work cycles

\begin{tabular}{|c|c|c|}
\hline \multirow[t]{2}{*}{ Actual } & \multicolumn{2}{|c|}{ Predicted } \\
\hline & Yes & No \\
\hline Yes & 35 (TP) & $4(\mathrm{FN})$ \\
\hline No & 8 (FP) & $35(\mathrm{TN})$ \\
\hline
\end{tabular}

Table 6. Quantitative summary of the labour input of the monitored steel fixing and electrical tasks

\begin{tabular}{|lccc|}
\hline Data sets & $\begin{array}{c}\text { GT labour } \\
\text { input }\end{array}$ & $\begin{array}{c}\text { Detected } \\
\text { labour input }\end{array}$ & Accuracy: \% \\
\hline $\begin{array}{l}\text { Worker '1' (data set } \\
\text { steel) }\end{array}$ & 29.71 & 26.05 & 88 \\
$\begin{array}{c}\text { Worker '2' (data set } \\
\text { electrical) }\end{array}$ & 20.30 & 15.64 & 77 \\
$\begin{array}{c}\text { Worker '3' (data set } \\
\text { electrical) } \\
\text { Average }\end{array}$ & 19.68 & 17.24 & 88 \\
\hline
\end{tabular}

can be monitored for multiple workers without any prior knowledge of the type of tasks the workers perform, $(b)$ workflow inefficiencies and potential management issues can be automatically identified by observing the automatically extracted 3D trajectories that correspond to abnormal work cycles without having to manually produce the so-called spaghetti diagrams that is, motion on the floor plane, (Nyström and Per, 2009) and (c) the hypothesis of this paper that task productivity of construction workers can be monitored through their trajectory data is true.

The research presented in this paper tried to address most of the challenges related to the monitoring of labour productivity for construction workers. However, there are still some limitations: (a) work cycles that depict waiting time - that is, workers simply standing without performing any task are also mistakenly detected as productive, $(b)$ the trajectory data were extracted from two cameras only and $(c)$ tasks that do not depict direct work - that is, are primarily characterised by motion (e.g. transferring materials), cannot be monitored effectively with the proposed method as in such cases, the 'move' events are those that depict the actual labour input instead of the 'stop' events; such tasks depict travel activities related to the transfer of materials/equipment and information. Future work will focus on $(a)$ posture analysis in order to better evaluate the waiting time, $(b)$ a task identifier that would allow the proposed method to also monitor tasks that are described by the 'move' events and (c) applying a computervision based method that requires less smoothing in order to avoid missing work cycles.

\section{Acknowledgments}

This research is an ICASE studentship award, supported by EPSRC and LAING O'ROURKE PLC under grant number 13440016. Any opinions, findings and conclusions or recommendations included in this paper are those of the authors and do not necessarily reflect the views of organisations and people mentioned above.

\section{REFERENCES}

Akhavian R and Behzadan AH (2016) Smartphone-based construction workers' activity recognition and classification. Automation in Construction 71(Part 2): 198-209, https://doi.org/10.1016/j.autcon. 2016.08.015.

AMAC Consultants (2004) Productivity Measurement and Analysis. The University of British Columbia, Vancouver, BC, Canada.

Bai Y, Huan J and Peddi A (2008) Development of Human Poses for the Determination of On-Site Construction Productivity in Real-time. University of Kansas Center for Research, Inc., Lawrence, KS, USA.

Boonstra AM, Fidler V and Eisma WH (1993) Walking speed of normal subjects and amputees: aspects of validity of gait analysis. Prosthetics and Orthotics International 17(2): 78-82, https://doi.org/10.3109/ 03093649309164360.

Bügler M, Ogunmakin G, Teizer J, Vela PA and Borrmann A (2014) A comprehensive methodology for vision-based progress and activity estimation of excavation processes for productivity assessment. In Proceedings of the 21st International Workshop: Intelligent Computing in Engineering. EG-ICE, Cardiff, UK, pp. 1-10.

Carrasco V, Hall B and Sweany J (2013) Denver International AirportSouth Terminal Redevelopment Program: Labor and Productivity Analysis. Denver International Airport, Denver, CO, USA.

Cheng CF, Rashidi A, Davenport MA and Anderson DV (2017) Activity analysis of construction equipment using audio signals and support vector machines. Automation in Construction 81: 240-253, https://doi. org/10.1016/j.autcon.2017.06.005.

Cheng T, Venugopal M, Teizer J and Vela PA (2011) Performance evaluation of ultra wideband technology for construction resource location tracking in harsh environments. Automation in Construction 20(8): 1173-1184, https://doi.org/10.1016/j.autcon.2011.05.001.

Cheng T, Teizer J, Migliaccio GC and Gatti UC (2013) Automated tasklevel activity analysis through fusion of real time location sensors and worker's thoracic posture data. Automation in Construction 29: 24-39, https://doi.org/10.1016/j.autcon.2012.08.003.

Dozzi SP and AbourRizk SM (1993) Productivity in Construction. Institute for Research in Construction, National Research Council, Ottawa, Canada.

El-Gohary KM and Aziz RF (2014) Factors influencing construction labor productivity in Egypt. Journal of Management in Engineering 30(1): 1-9, https://doi.org/10.1061/(ASCE)ME.1943-5479.0000168.

Eng J (2003) Sample size estimation: how many individuals should be studied? Radiology 227(2): 309-313, https://doi.org/https://pubs.rsna. org/doi/full/10.1148/radiol.2272012051.

European Construction Industry Federation (2019) Annual Report. See http://www.fiec.eu/en/search-73.aspx?q=annual report (accessed 20/08/2020).

Gatti UC, Migliaccio GC, Bogus SM and Schneider S (2014) An exploratory study of the relationship between construction workforce physical strain and task level productivity. Construction Management and Economics 32(6): 548-564, https://doi.org/10.1080/01446193.2013. 831463.

Gong J and Caldas CH (2011) An object recognition, tracking, and contextual reasoning-based video interpretation method for rapid productivity analysis of construction operations. Automation in Construction 20(8): 1211-1226, https://doi.org/10.1016/j.autcon.2011. 05.005 .

Gor RM and Man M (2009) Chapter 6: forecasting techniques. Industrial Statistics and Operational Management. See http://nsdl.niscair.res.in/ jspui/browse?type $=$ authorandvalue $=$ Gor $\% 2 \mathrm{C}+$ Ravi + Mahendra . 
Guiñón JL, Ortega E, García-antón J and Pérez-Herranz V (2007) Moving average and Savitzki-Golay smoothing filters using Mathcad. In International Conference on Engineering Education - ICEE. University of Coimbra, Coimbra, Portugal, pp. 1-4.

Gundecha MM (2012) Study of Factors Affecting Labor Productivity at a Building Construction Project in the USA: Web Survey. MS thesis, North Dakota State University of Agriculture and Applied Science, Fargo, ND, USA.

Hanna AS, Taylor CS and Sullivan KT (2005) Impact of extended overtime on construction labor productivity. Journal of Construction Engineering and Management 131(6): 734-739, https://doi.org/10. 1061/(ASCE)0733-9364(2005)131:6(734).

Hartley RI (1997) In defence of the 8-point algorithm. IEEE Transactions on Pattern Analysis and Machine Intelligence 19(6): 1064-1070, https://doi.org/10.1109/34.601246.

Horman MJ and Kenley R (2005) Quantifying levels of wasted time in construction with meta-analysis. Journal of Construction Engineering and Management 131(1): 52-61, https://doi.org/10.1061/(ASCE)07339364(2005)131:1(52).

Jarkas AM and Bitar CG (2012) Factors affecting construction labor productivity in Kuwait. Journal of Construction Engineering and Management 138(7): 811-820, https://doi.org/10.1061/(ASCE)CO. 1943-7862.0000501.

Jiang $\mathrm{H}$, Lin $\mathrm{P}$, Qiang $\mathrm{M}$ and Fan Q (2015) A labor consumption measurement system based on real-time tracking technology for dam construction site. Automation in Construction 52: 1-15, https://doi.org/ 10.1016/j.autcon.2015.02.004.

Juels A (2006) RFID security and privacy: a research survey. IEEE Journal on Selected Areas in Communications 24(2): 381-394, https:// doi.org/10.1109/JSAC.2005.861395.

Khosrowpour A, Fedorov I, Holynski A, Niebles C and Golparvar-Fard M

(2014) Automated worker activity analysis in indoor environments for direct-work rate improvement from long sequences of RGB-D Images. In Construction Research Congress 2014. Atlanta, GA, USA, pp. 140-149.

Konstantinou E and Brilakis I (2018a) Trajectory-based worker task productivity monitoring. In International Symposium on Automation and Robotics in Construction. International Association for Automation and Robotics in Construction, Berlin, Germany, pp. $1145-1151$.

Konstantinou E and Brilakis I (2018b) Matching construction workers across views for automated $3 \mathrm{D}$ vision tracking on-site. Journal of Construction Engineering and Management 144(7): 1-12, https://doi. org/10.1061/(ASCE)CO.1943-7862.0001508.

Konstantinou E, Lasenby J and Brilakis I (2019) Adaptive computer vision-based 2D tracking of workers in complex environments. Automation in Construction 103: 168-184, https://doi.org/10.1016/j. autcon.2019.01.018.

Kuykendall CJO (2007) Key Factors Affecting Labor Productivity in the Construction Industry. MS thesis, University of Florida, Gainesville, FL, USA.

Lim E and Alum J (1995) Construction productivity: issues encountered by contractors in Singapore. International Journal of Project Management 13(1): 51-58, https://doi.org/10.1016/0263-7863(95) 95704-H.

Lim EC (1996) The Analysis of Productivity in Building Construction. Doctoral thesis. Loughborough University, Loughborough, UK.

Makulsawatudom A, Emsley M and Sinthawanarong K (2004) Critical factors influencing construction productivity in Thailand. International Journal of Applied Science and Technology 14(3): 1-6.

Nasr E, Shehab T and Vlad A (2013) Tracking systems in construction: applications and comparisons. In 49th ASC Annual International Conference Proceedings, 9-13 April 2013, San Luis Obispo, CA, USA.

Navon R and Goldschmidt E (2003) Can labor inputs be measured and controlled automatically? Journal of Construction Engineering and
Management 129(4): 437-445, https://doi.org/10.1061/(ASCE)07339364(2003)129:4(437).

Nyström D and Per S (2009) Productivity Increase Valve and Pipe Assembly: An Investigation of How to Improve the Manufacturing Process in a Large Variant Production Environment. Master thesis. Chalmers University of Technology, Göteborg, Sweden.

Picard HE (2004) Driving down construction project labor cost. In Second International Conference on Structural and Construction Engineering (Bontempti F (ed.)). AA Balkema, Lisse, the Netherlands, vol. 1, pp. 269-273.

Sedehi AJ (2010) Leveraging Radio Frequency Technology Identification for Productivity Analysis in High-Rise Construction. MS thesis, Georgia Institute of Technology, Atlanta, GA, USA.

Shehata ME and El-Gohary KM (2011) Towards improving construction labor productivity and projects' performance. Alexandria Engineering Journal 50(4): 321-330, https://doi.org/10.1016/j.aej.2012.02.001.

Teicholz P (2004) Labor productivity declines in the construction industry: causes and remedies. AECbytes Viewpoint \#4, 14 April. See http://www.aecbytes.com/viewpoint/2004/issue_4.html (accessed 20/ 08/2020).

Teicholz P (2013) Labor-productivity declines in the construction industry: causes and remedies (another look). AECbytes, 14 March. See http://www.aecbytes.com/viewpoint/2013/issue_67.html (accessed 20/08/2020).

Weerasinghe IPT and Ruwanpura JY (2010) Automated multiple objects tracking system (AMOTS). In Construction Research Congress. Banff, Alberta, Canada, pp. 11-20.

Zhang Z (2000) A flexible new technique for camera calibration. IEEE Transactions on Pattern Analysis and Machine Intelligence 22(11): 1330-1334, https://doi.org/10.1109/34.888718.

Zou J and Kim H (2007) Using hue, saturation, and value color space for hydraulic excavator idle time analysis. Journal of Computing in Civil Engineering 21(4): 238-246, https://doi.org/10.1061/(ASCE)08873801(2007)21:4(238).

\section{How can you contribute?}

To discuss this paper, please email up to 500 words to the editor at journals@ice.org.uk. Your contribution will be forwarded to the author(s) for a reply and, if considered appropriate by the editorial board, it will be published as discussion in a future issue of the journal.

Proceedings journals rely entirely on contributions from the civil engineering profession (and allied disciplines). Information about how to submit your paper online is available at www.icevirtuallibrary.com/page/authors, where you will also find detailed author guidelines. 